\title{
Phase separation in suspensions of colloids, polymers and nanoparticles: Role of solvent quality, physical mesh, and nonlocal entropic repulsion
}

\author{
Y.-L. Chen and K. S. Schweizer ${ }^{\mathrm{a})}$ \\ Departments of Chemical Engineering and Materials Science and Engineering, University of Illinois, \\ Urbana, Illinois 61801 \\ M. Fuchs \\ Institut Charles Sadron, 6 rue Boussingault, 67083 Strasbourg Cedex, France
}

(Received 28 October 2002; accepted 22 November 2002)

\begin{abstract}
Analytic and numerical microscopic integral equation theory for polymer-particle suspensions is employed to investigate the dependence of fluid-fluid phase separation on size asymmetry, solvent quality, and higher order polymer-polymer interactions. For athermal good solvents, our prior novel prediction of enhanced miscibility with increasing (decreasing) polymer (particle) size is found not to be fundamentally tied to physical mesh formation or strong polymer-induced colloid clustering. Rather, the key is a proper treatment of the polymer second virial coefficient, which is sensitive to how chains organize in the empty space between particles. The origin of the qualitative error made by classic mean-field theories for the shifting of phase boundaries with size asymmetry is established. The phase separation behavior predicted by integral equation theory for ideal polymers is completely different than the athermal case for all size asymmetries and particle volume fractions, thereby establishing the remarkably large consequences of polymer-polymer repulsions. For large polymers or small nanoparticles under ideal solvent conditions, the suspension miscibility worsens with increasing size asymmetry, opposite to the athermal solvent behavior. However, over a significant range of intermediate size asymmetries the spinodal curves are either nearly constant, or display a nonmonotonic shifting, as size asymmetry is varied. Higher order contributions in polymer concentration modestly stabilize the miscible phase in both athermal and ideal solvents.
\end{abstract}

\section{INTRODUCTION}

The phase behavior of polymer-particle suspensions is a complex subject due to the large variability in polymer/ particle size disparity, solvency conditions, and the presence of multiple physical forces including Coulomb, excluded volume, and attractive van der Waals. ${ }^{1-7}$ The simplest realization of such ternary mixtures is the (nearly) athermal system composed of flexible nonadsorbing polymer coils (radius-of-gyration $R_{g}$ ) and hard sphere colloids (radius $R$ ). Such a model suspension continues to display rich and puzzling behavior as the full parameter space is explored, and can exist as a homogeneous fluid, two coexisting fluids, coexisting crystal and fluid, or in nonequilibrium gel or glass states. $^{2-6}$ Relevant parameters are polymer-particle size asymmetry ratio $\xi=R_{g} / R$, particle volume fraction $\phi_{s}$, polymer concentration in units of the dilute-semidilute crossover value $c_{p} / c_{p}^{*}$, and solvent quality (good, theta, poor) which controls the effective polymer-polymer interactions. $^{2-4,7-11}$

For $R_{g} / R>0.3$ or so, such suspensions first undergo fluid-fluid phase separation with increasing $c_{p} / c_{p}^{*}$ and/or particle volume fraction. ${ }^{2-4,7,11}$ This is the topic of the present work. Demixing is driven by the entropic "depletion attraction" between particles induced by polymer additives. ${ }^{12}$ Systematic experimental study of the dependence of phase

${ }^{a)}$ Electronic mail: kschweiz@uiuc.edu separation on size asymmetry has only recently been accomplished under athermal good solvent conditions characterized by strong repulsive interactions between all components. ${ }^{4}$ Classic theories ${ }^{7,11,12}$ have been formulated from a colloidal perspective $\left(R \gg R_{g}\right) \quad$ utilizing multiple simplifications, the most severe of which are: (i) approximating a flexible polymer by a sphere which neglects internal or segmental (conformational) degrees of freedom, and (ii) ignoring all polymer-polymer interactions (polymer $\approx$ "phantom sphere"). These approximations are plausible (at best) under the restrictive conditions of an ideal theta solvent, dilute polymer concentrations $\left(c_{p} / c_{p}^{*} \ll 1\right)$, and the $R>R_{g}$ colloidal regime. Based on these simplifications, the classic statistical thermodynamic approaches (two-component ${ }^{7}$ or effective one-component ${ }^{11,12}$ ) predict, in analogy with the liquid-vapor transition of a one-component fluid, that suspensions become less miscible as $R_{g} / R$ increases due to the growing spatial range of the depletion attraction. The domain of validity of this prediction has been unknown due to the difficulties in relaxing simplifications (i) and (ii), which require an explicit treatment of polymer statistics. ${ }^{13}$ Recently, a new microscopic liquid state description of structure, thermodynamics, and phase separation has been developed and applied ${ }^{8-10}$ which combines and extends ideas from atomic and polymeric integral equation theory. ${ }^{14-16}$ This "polymer reference interaction site model" (PRISM) theory ${ }^{14,15}$ is potentially applicable over all ranges of suspension parameters. Initial applications to athermal 
mixtures resulted in the novel prediction that suspension miscibility improves with increasing $R_{g} / R$, opposite to the classic theories. ${ }^{7,11}$

The qualitative differences between the classic and liquid state theory phase behavior predictions motivated Ramakrishnan et al. $^{4}$ to experimentally study model athermal suspensions where $R_{g} / R$ was varied from 0.026 to 1.4 . Miscibility was found to monotonically improve with increasing $R_{g} / R$, confirming the novel PRISM prediction. This trend for $R_{g}<R$ was subsequently found via large scale Monte Carlo simulation using an approximate "soft colloid" model of polymers. ${ }^{17}$ Turbidity measurements of colloidal concentration fluctuations in the homogeneous fluid phase have also been performed. ${ }^{18}$ PRISM theory was found to be quite accurate over the entire range of size asymmetry, while the classic approaches incurred large errors for both small and large values of $R_{g} / R$.

A primary conclusion drawn from the theoryexperiment comparisons $s^{4,7,10,18}$ is that in athermal suspensions the consequences of relaxing approximations (i) and (ii) are very large. However, a "simple" and unique explanation is not readily deduced since PRISM theory includes multiple effects not in the classic approaches: (a) nonlocal polymer-particle entropic repulsion; (b) polymer-polymer repulsions; (c) polymer conformational degrees of freedom; and (d) nondilute polymer concentration effects. Disentanglement of the consequences of including these aspects is not straightforward.

The goals of the present paper are to establish the fundamental origin of the novel phase separation prediction of PRISM theory by systematically simplifying it. In pursuit of this goal, three additional questions are addressed. (1) The role of physical mesh formation and polymer-induced colloidal clustering (free-volume modification) in an athermal suspension is investigated by taking the dilute polymer concentration limit in analogy with what is done a priori in the classic approaches. Physically, one expects such a simplification will enhance depletion attraction between particles and facilitate phase separation, but in a manner that will depend on $R_{g} / R$ and colloid volume fraction. (2) The direct and indirect role of polymer-polymer repulsions is investigated by considering the antithesis of an athermal solvent: an "ideal theta" solvent where polymers are treated as noninteracting Gaussian random coils. Such ideal models have been the primary focus of prior theoretical ${ }^{7,11,12,19-21}$ and computer simulation $^{22}$ studies due to the simplifications it affords. The absence of polymer-polymer repulsions is expected to enhance depletion attraction since no excess freeenergy penalty is incurred if polymers cluster. (3) The importance of the loss of conformational entropy when polymer segments are close to hard particles is established under ideal solvent conditions. Inclusion of this "nonlocal entropy" effect is the primary new component of the recent PRISM theory, ${ }^{8-10}$ and it plays a critical role in the novel phase separation predictions for athermal suspensions. Although any extra polymer-particle repulsion favors phase separation, the strongly nonadditive nature of excluded volume interactions in an ideal solvent is expected to render their effects of a quantitative, not qualitative, nature.
The above discussion provides a summary of many of our findings which will be established using a combination of analytic analysis and specific numerical calculations. Several unexpected subtleties also arise for phase separation in theta solvents. We also establish the origins of the errors of the classic approaches which become increasingly severe as colloid volume fraction increases and/or the $R_{g}>R$ regime is entered.

The remainder of the paper is structured as follows. In Sec. II the two classes of theories and models considered are described. Since the technical details of the theories have been thoroughly documented in the literature, ${ }^{7-10}$ we only summarize the key elements. The dilute polymer version of PRISM theory is developed in Sec. III and contrasted with the full theory and the classic mean-field approach. Section IV presents our numerical and analytic results and addresses points (1)-(3) enumerated above. The paper concludes in Sec. V with a summary and future outlook. Technical details of the numerical implementation of PRISM theory are given in the Appendix.

\section{THEORY AND MOLECULAR MODELS}

A ternary mixture of polymers, particles, and small molecule solvent is simplified to an effective binary system by treating the solvent as a continuum which influences only the effective pair decomposable interactions between polymer segments and particles. The relevant system parameters are: coil radius of gyration $R_{g}$, colloid diameter $D=2 R$, polymer number density $c_{p}$, and colloid volume fraction $\phi_{s}$ $=\pi \rho_{s} D^{3} / 6$. The two binary mixture theories considered are the "phantom sphere free-volume" (PSFV) thermodynamic approach $^{7}$ and PRISM. ${ }^{8-10}$

\section{A. PSFV approach}

The phantom sphere free-volume approach of Lekkerkerker and co-workers ${ }^{7}$ considers a model suspension of hard spheres and noninteracting polymer coils in the dilute polymer limit. Polymers chains are replaced by spheres with no internal (conformational) degrees of freedom which can pass freely through each other ("ideal") but act as hard spheres of radius $R_{g}$ when interacting with colloids. The Helmholtz free energy consists of three terms: a pure hard sphere (HS) part, a pure (noninteracting) polymer contribution due to center-of-mass translation, and a polymer-colloid interaction term strictly linear in $c_{p}$. The latter follows from computing the free-energy cost to insert a single polymer into the hard sphere fluid and is quantified by a "free-volume fraction," $\alpha$, which depends on $\phi_{s}$ and size asymmetry ratio $\xi=R_{g} / R$.

Using scaled particle theory (SPT) for hard sphere mixtures, ${ }^{23}$ the insertion chemical potential, $\beta \delta \mu_{p}$, and fractional free volume, $\alpha$, are given by

$$
\begin{aligned}
& \beta \delta \mu_{p}=-\ln \alpha=-\ln \left(1-\phi_{s}\right)+A \frac{\phi_{s}}{1-\phi_{s}}+B\left(\frac{\phi_{s}}{1-\phi_{s}}\right)^{2} \\
&+C\left(\frac{\phi_{s}}{1-\phi_{s}}\right)^{3} \\
& A=3 \xi+3 \xi^{2}+\xi^{3}, \quad B=9 \xi^{2} / 2+3 \xi^{3}, \quad C=3 \xi^{3}
\end{aligned}
$$


from which the phase boundaries may be determined. ${ }^{7}$ The spinodal curve, defined as the polymer and colloid densities at which the osmotic compressibility diverges, is

$$
\begin{aligned}
& \frac{c_{p}^{+}}{c_{p}^{*}}=\frac{\left(1-\phi_{s}\right)^{4}\left(1+2 \phi_{s}\right)}{\Lambda} \\
& \Lambda=\phi_{s}\left[-12 t_{1}^{3}+15 \xi t_{1}^{2} t_{2}-6 \xi^{2} t_{1} t_{2}^{2}+\xi^{3} t_{2}^{3}\right],
\end{aligned}
$$

where $t_{1}=\phi_{s}-1, t_{2}=2 \phi_{s}+1, c_{p}^{+}$is the polymer concentration at the spinodal, and $1 / c_{p}^{*}=4 \pi R_{g}^{3} / 3$ is the polymer coil volume $V_{p}$. The PSFV theory predicts fluid-fluid spinodal (and binodal) boundaries shift monotonically to smaller reduced polymer concentration $c_{p} / c_{p}^{*}$ as $\xi$ increases. Even in the $\xi<1$ "colloid" regime under relatively dilute polymer concentrations, this is the opposite trend observed in recent athermal good solvent experiments. ${ }^{4}$

\section{B. PRISM theory}

PRISM theory ${ }^{14,15}$ is a macromolecular extension of the RISM theory of Chandler and Andersen for small, rigid, molecular fluids. ${ }^{16}$ It is based on an interaction site representation of molecules and a generalization of the matrix Ornstein-Zernike equation. The latter relates the intermolecular site-site pair correlation functions as a function of scalar site separation $h_{i j}(r)=g_{i j}(r)-1$, the intermolecular site-site direct correlation functions $C_{i j}(r)$, and the intramolecular structure factors $\hat{\omega}_{i}(k)$, where the caret denotes Fourier transformed functions (wave vector, $k$ ). The subscripts $i$ and $j$ denote the mixture species, and $r$ is the real space separation. In Fourier space, the matrix integral equations for molecules composed of chemically identical interaction sites are

$$
\hat{h}_{i j}(k)=\hat{\omega}_{i}(k)\left[\hat{C}_{i j}(k) \hat{\omega}_{j}(k)+\sum_{l} \hat{C}_{i l}(k) \rho_{l} \hat{h}_{l j}(k)\right] .
$$

Explicit chain end effects have been ignored. ${ }^{14}$ The structure factors describing collective partial density fluctuations are given by

$$
\hat{S}_{i j}(k)=\rho_{i} \hat{\omega}_{i}(k) \delta_{i j}+\rho_{i} \rho_{j} \hat{h}_{i j}(k) .
$$

The diagonal elements of the matrix of partial structure factors are nondimensionalized via division by $\rho_{i}$, the site number density $\left(\rho_{s}, \rho_{p}=c_{p} N\right)$. The intramolecular structure factor for hard sphere colloids is $\hat{\omega}_{s}(k)=1$. A field theoretic type description of flexible polymers as infinitely thin continuous Gaussian random walks or "threads" is employed. ${ }^{14}$ In this model, the number of sites per polymer $N$ is irrelevant and only the global polymer size enters. ${ }^{8-10}$ The intramolecular structure factor is approximated by

$$
\widetilde{\omega}_{p}(k)=\frac{1}{N} \hat{\omega}_{p}(k)=\frac{1}{1+k^{2} R_{g}^{2} / 2},
$$

which is accurate to within $15 \%$ of the exact Gaussian model $^{24}$ and simplifies the partial analytic solution of the coupled nonlinear integral equations. ${ }^{25}$ This form is rigor- ously applicable for "ideal" polymers. We also employ it as an approximate "effective" Gaussian model for interacting polymers as commonly done in field theoretic approaches. ${ }^{24}$

To solve Eq. (3), the new "modified Percus-Yevick" (mPY) closure approximation for hard core site-site intermolecular interactions is employed. ${ }^{8-10}$ The mPY closure enforces the local exclusion constraint and accounts for nonlocal polymer-particle entropic effects in a thermodynamically consistent manner, and is given by

$$
\begin{aligned}
& g_{i i}(r)=0 \text { for } r<R_{i i} \\
& C_{i i}(r) \cong 0 \text { for } r>R_{i i} \\
& \hat{C}_{p s}(k)=\frac{\hat{C}_{p s}^{s}(k)}{1+k^{2} \lambda^{2}}, \quad C_{p s}^{s}(r) \cong 0 \text { for } r>R_{p s},
\end{aligned}
$$

where $R_{i j}$ is the distance of closest approach between interaction sites of the type $i$ and $j\left(R_{s s}=D\right.$ and $\left.R_{p s}=D / 2\right)$. The correlation length $\lambda$ describes the loss of conformational entropy when polymer segments are within a distance $\lambda$ of a particle surface. It is computed by enforcing the constraint of thermodynamic self-consistency on the polymer insertion chemical potential based on the compressibility and freeenergy routes to the thermodynamics. ${ }^{8-10}$ If $\lambda=0$, the sitesite PY approximation is recovered. In analogy with hard sphere mixtures, ${ }^{26,27}$ based on the PY closure, spinodal phase separation is not found in athermal mixtures of repelling coils and spheres due to the quantitative underestimate of depletion attraction effects by the local PY approximation. $8,10,28$

The nonlocality length $\lambda$ is a function of all system variables, and depends on $c_{p}$ and $\phi_{s}$ in a manner that captures many-body screening effects due to polymer and colloid concentration fluctuations. ${ }^{8-10}$ A convenient analytic interpolation formula for $\lambda$ is ${ }^{9}$

$$
\begin{aligned}
\lambda^{-1} & =\frac{\sqrt{2}+2 \pi c_{p} R_{g}^{3}}{R_{g}}+\frac{1+2 \phi_{s}}{1-\phi_{s}} \frac{\gamma_{1}}{D}, \text { athermal solvent } \\
\lambda^{-1} & =\frac{\sqrt{2}}{R_{g}}+\frac{1+2 \phi_{s}}{1-\phi_{s}} \frac{\gamma_{1}}{D}, \quad \text { ideal solvent }
\end{aligned}
$$

where $\gamma_{1} \equiv 1+\sqrt{5}$. The athermal solvent expression incurs errors in rigorous enforcement of thermodynamic consistency of $<20 \%$ over the entire parameter range. The ideal solvent formula follows from the athermal result by ignoring blob screening effects consistent with the literal neglect of polymer-polymer interactions. ${ }^{10,13}$

For the thread model under athermal good solvent conditions, the pointlike polymer-polymer repulsions enter via the PY closure: ${ }^{14} C_{p p}(\mathbf{r}=\mathbf{0})=C_{p p} \delta(\mathbf{r})$, where $C_{p p}$ is chosen to enforce the local segmental scale excluded volume condition, $g_{p p}(r=0)=0$, and is necessarily a function of all system parameters. In field theoretic language, $-C_{p p}$ plays the role of a self-consistently determined excluded volume parameter. In strong contrast, ideal solvent conditions are defined as allowing polymers to pass through each other, $C_{p p}(\mathbf{r})=0$ for all $\mathbf{r}$. In the absence of particles and in the 
dilute polymer limit, $C_{p p}=0$ corresponds to the classic definition of ideal theta conditions and a vanishing second (and higher order) virial coefficient. ${ }^{29}$ Analytic progress has previously been made for the athermal case based on the Wiener-Hopf factorization method. ${ }^{9}$ In the present work we also employ iterative numerical methods as described in the Appendix.

The criterion for fluid-fluid spinodal phase separation is the divergence at $k=0$ of all partial structure factors, or equivalently vanishing of the determinant of the free-energy second partial derivative matrix. This follows from Eqs. (3) and (4) as ${ }^{14}$

$$
0=1-\rho_{s} \hat{C}_{s s}-c_{p} \widetilde{C}_{p p}+c_{p} \rho_{s}\left(\widetilde{C}_{p p} \hat{C}_{s s}-\widetilde{C}_{p s}^{2}\right),
$$

where $\hat{C}_{i j}=\hat{C}_{i j}(k=0), \widetilde{C}_{p p}=\hat{C}_{p p} N^{2}$, and $\widetilde{C}_{p s}=\hat{C}_{p s} N$.

The single polymer insertion chemical potential calculated via the compressibility route is given by ${ }^{9}$

$$
\left.N \beta \delta \mu_{p}\right|_{c_{p}=0}=-\int_{0}^{\rho_{s}} \widetilde{C}_{p s}\left(k=0 ; \rho_{s}^{\prime}\right) d \rho_{s}^{\prime} .
$$

For this property, solvent quality as manifested in the effective intermolecular polymer-polymer interaction is irrelevant. Analytic analysis of PRISM-mPY yields an expression that reduces in the extreme colloid $(\xi \rightarrow 0)$ and extreme nanoparticle $(\xi \rightarrow \infty)$ limits to 9

$$
\begin{aligned}
& \left.N \beta \delta \mu_{p}\right|_{c_{p}=0}=-\ln \left(1-\phi_{s}\right), \quad \xi \rightarrow 0, \\
& \left.N \beta \delta \mu_{p}\right|_{c_{p}=0}=\frac{3}{8} \gamma_{1} \xi^{2} \frac{\phi_{s}\left(2+\phi_{s}\right)}{\left(1-\phi_{s}\right)^{2}}, \quad \xi \rightarrow \infty .
\end{aligned}
$$

For large, Gaussian polymers, Eq. (11) correctly scales as $\left(R_{g} / R\right)^{d_{F} \sim N} \sim$, where $d_{F}=2$ is the fractal dimension of an ideal random coil. This is in strong contrast to the $\sim\left(R_{g} / R\right)^{3}$ scaling in Eq. (1) of the PSFV theory ${ }^{7}$ which becomes unphysical as $\xi>1$, since the chain nature of polymers allows them to "wrap around" colloidal obstacles. ${ }^{8}$

\section{PERTURBATIVE LIMIT}

PRISM-mPY theory accounts for contributions through all orders in polymer concentration; hence, "physical mesh" formation, $9,10,24$ as the semidilute solution state is approached and entered. A priori, it is not obvious how important this aspect is. In order to elucidate the connection of the integral equation theory to the PSFV model, and the primary physical origin of the novel predictions of the former for athermal solvents, we analyze PRISM theory through lowest order in polymer concentration. The analytic analysis provides the basis for interpretation of the numerical results presented in Sec. IV.

\section{A. Rigorous lowest order analysis}

We first consider an exact perturbative in polymer concentration analysis, independent of solvent quality. Inspection of the spinodal condition of Eq. (8) reveals that through lowest order only the infinite dilution values of $\widetilde{C}_{p s}$ and $\widetilde{C}_{p p}$ are needed, corresponding to the problems of one or two polymers in a hard sphere fluid. These can be computed from Eq. (3), which decouple into three independent equations. However, the first-order correction to the colloid-colloid direct correlation function, which describes polymer-induced modification of sphere packing, is required. Thus, through lowest order

$$
\begin{aligned}
& \widetilde{C}_{p p}\left(c_{p}\right)=\widetilde{C}_{p p}\left(c_{p} \rightarrow 0\right) \doteq \widetilde{C}_{p p}^{0}, \\
& \hat{C}_{s s}\left(c_{p}\right)=\hat{C}_{s s}\left(c_{p} \rightarrow 0\right)+c_{p} \Delta \hat{C}_{s s} \doteq \hat{C}_{\mathrm{HS}}+c_{p} \Delta \hat{C}_{s s}, \\
& \widetilde{C}_{p s}\left(c_{p}\right)=\widetilde{C}_{p s}\left(c_{p} \rightarrow 0\right) \doteq \widetilde{C}_{p s}^{0},
\end{aligned}
$$

which is referred to as $\mathrm{O}\left(c_{p}\right)$ PRISM, or "PRISM0," theory. The quantity $\Delta C_{s s}$ follows from a perturbative analysis of Eq. (3) and is given by the linear integral equation

$$
\begin{aligned}
& \Delta \hat{h}_{s s}(k)=\hat{S}_{\mathrm{HS}}^{2}(k)\left[\Delta \hat{C}_{s s}(k)+\widetilde{\omega}_{p}(k) \widetilde{C}_{p s}^{0}(k)^{2}\right], \\
& \Delta h_{s s}(r)=0, \quad r<2 R, \quad \Delta C_{s s}(r)=0, \quad r>2 R,
\end{aligned}
$$

where $\hat{S}_{\mathrm{HS}}(k)=\left(1-\rho_{S} \hat{C}_{\mathrm{HS}}(k)\right)^{-1}$ is the hard sphere structure factor, and $\hat{S}_{\mathrm{HS}}(k=0)=\left(1-\phi_{s}\right)^{4} /\left(1+2 \phi_{s}\right)^{2}$ is the dimensionless compressibility. ${ }^{30}$ Analytic expressions are available for $\widetilde{C}_{p p}^{0}$ and $\widetilde{C}_{p s}^{0},{ }^{9}$ but not $\Delta \hat{C}_{s s}$.

Through leading order in polymer concentration Eq. (13) applies independent of intermolecular polymer-polymer interactions. Thus, within our effective Gaussian chain model approximation, solvent quality (interchain interactions) plays no role in polymer mediated (depletion) colloidal clustering through lowest order. The spinodal boundary in Eq. (8) may then be rigorously written through $\mathrm{O}\left(c_{p}\right)$ as

$$
0=1-c_{p} \rho_{s} \hat{S}_{\mathrm{HS}} \Delta \hat{C}_{s s}+2 c_{p} B_{2, p p} .
$$

Here, the polymer molecule effective second virial coefficient consists of "direct" and "indirect" (colloid mediated) contributions 9,28

$$
\begin{gathered}
2 B_{2, p p}=-\hat{h}_{p p}^{0} N^{2}=-\widetilde{C}_{p p}^{0}-\rho_{s} \hat{S}_{\mathrm{HS}} \widetilde{C}_{p s}^{02}, \\
\frac{c_{p}^{+}}{c_{p}^{*}}=\frac{V_{p}}{-2 B_{2, p p}+\rho_{s} \hat{S}_{\mathrm{HS}} \Delta \hat{C}_{s s}} \\
=\frac{V_{p}}{\widetilde{C}_{p p}^{0}+\rho_{s} \hat{S}_{\mathrm{HS}}\left(\Delta \hat{C}_{s s}+\widetilde{C}_{p s}^{0}{ }^{2}\right)},
\end{gathered}
$$

where $c_{p}^{+}$is the polymer concentration at the spinodal. Note that polymer-polymer interactions or solvent quality is relevant to demixing via its influence on $B_{2, p p}$, which will be shown to be very important for phase behavior.

An alternative exact form of the spinodal boundary follows from using the generalized Maxwell relation ${ }^{31}$ appropriate to the $c_{p} \rightarrow 0$ limit of present interest

$$
\Delta \hat{C}_{s s}=\partial \hat{C}_{s s} / \partial \rho_{p}=-\partial^{2} \delta \mu_{p} / \partial \rho_{s}^{2} .
$$


Combining Eqs. (14) and (17) yields

$$
\begin{aligned}
& 0=1+2 c_{p} B_{2, p p}+c_{p} \rho_{s} \hat{S}_{\mathrm{HS}} \partial^{2} \beta \delta \mu_{p} / \partial \phi_{s}^{2}, \\
& \frac{c_{p}^{+}}{c_{p}^{*}}=\left(-\frac{2 B_{2, p p}}{V_{p}}-\phi_{s}\left(\frac{R}{R_{g}}\right)^{3} \hat{S}_{\mathrm{HS}} \frac{\partial^{2}}{\partial \phi_{s}^{2}} \beta \delta \mu_{p}\right)^{-1} .
\end{aligned}
$$

The second term in the right-hand side denominator of Eq. (18) is negative. Hence, phase separation requires a sufficiently negative $B_{2, p p}$ corresponding to colloid driven clustering of polymer coils. This is always possible under ideal coil conditions where excluded volume effects are strongly nonadditive, since polymers can (unphysically) pass through each other. But, it is not guaranteed for athermal solvents, making theoretical description of this case far more subtle and difficult.

The second term in Eq. (18) is unimportant if $\xi \gg 1$ [as rigorously follows from Eq. (20) below], and Eq. (18) takes the form appropriate for a polymer solution at an effective second virial level

$$
0=1+2 c_{p} B_{2, p p}, \quad \xi \gg 1 .
$$

For an athermal suspension, addition of polymer does not $a$ priori guarantee a negative $B_{2, p p}$ or fluid-fluid demixing.

In general, the qualitative behavior of the $\mathrm{O}\left(c_{p}\right)$ phase boundaries depends on the bare interactions and all three direct correlation functions. Further simplifications will be deduced by utilizing analytic "scaling" results in the extreme size asymmetry regimes

$$
\begin{aligned}
& \widetilde{C}_{p s}^{0} \sim-R_{g}^{2} R, \quad \Delta \hat{C}_{s s} \sim-R_{g}^{2} R^{4} \quad \text { for } \xi \gg 1, \\
& \widetilde{C}_{p s}^{0} \sim-R^{3}, \quad \Delta \hat{C}_{s s} \sim-R^{6} \text { for } \xi \ll 1 .
\end{aligned}
$$

These results follow from Eqs. (9) -(11) and (17), and have been discussed previously. ${ }^{9}$ Note that in the $\xi \gg 1$ extreme nanoparticle regime, $\Delta \hat{C}_{s s}$ is smaller than $\widetilde{C}_{p s}^{0}{ }^{2}$ by a factor $\sim \xi^{2}$, which provides the justification for the reduction of Eq. (18) to Eq. (19). Physically, it also implies that when polymers are much larger than the particles the local colloidal structure is nearly unaffected by the (now long-range) depletion attraction, ${ }^{10}$ consistent with classic van der Waals fluid ideas. ${ }^{14,16,30}$

The above analysis holds for arbitrary solvent quality. We now apply the perturbative results to understand the limiting ideal and athermal solvent behaviors.

\section{B. Ideal polymer limit}

In the ideal limit the polymer-polymer direct correlation function vanishes. Only the second (attractive) contribution in Eq. (15) remains. Employing Eq. (9) then yields

$$
2 B_{2, p p} \rightarrow-\rho_{s} \hat{S}_{\mathrm{HS}}\left(\frac{\partial \beta \delta \mu_{p}}{\partial \rho_{s}}\right)^{2}, \quad \text { ideal. }
$$

If polymers do not interact, the colloid-induced contribution to their second virial coefficient is determined solely by knowledge of the polymer insertion chemical potential and hard sphere compressibility. This simplification is assumed by PSFV theory ${ }^{7}$ and other recent approaches ("superposition approximation"). ${ }^{32}$ This result is not rigorous if polymers interact, and the consequences in good solvents are enormous. $^{4,10}$

Under ideal conditions, substituting Eq. (21) in Eq. (18) yields a spinodal condition which is applicable for all size asymmetry ratios and is identical in general form to that employed in the PSFV theory. The first and second derivatives of the polymer insertion chemical potential enter, which can be compactly expressed in terms of the fractional freevolume function, $\alpha=\exp \left(-\beta \delta \mu_{p}\right)$, as ${ }^{3,4}$

$$
\frac{c_{p}^{+}}{c_{p}^{*}}=\left(\phi_{s} \hat{S}_{\mathrm{HS}}\right)^{-1}\left(R_{g} / R\right)^{3} \alpha\left(\frac{\partial^{2} \alpha}{\partial \phi_{s}^{2}}\right)^{-1}, \quad \text { ideal. }
$$

This equation reduces exactly to the PSFV result of Eq. (2) if the ideal polymer coils are approximated as hard spheres and scaled particle theory is employed to compute the fractional free volume. Hence, for this ideal polymer limit the differences between the perturbative PRISM and PSFV theories can be precisely identified as entering solely through the single polymer insertion chemical potential.

In the $\xi \gg 1$ nanoparticle limit, Eqs. (15), (18), and (20) show that the colloid-induced attractive contribution to the polymer second virial coefficient dominates the determination of the spinodal. Thus, very large differences between PRISM and the PSFV theories occur due to the PSFV treatment of a polymer as a hard sphere. In particular, Eqs. (1), (11), and (22) yield

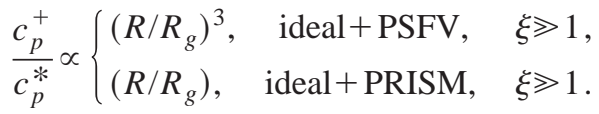

The "polymer $\approx$ hard sphere" approximation results in a massive reduction of miscibility, as also found more recently by an alternative approach. ${ }^{33}$

In the $\xi \ll 1$ colloid regime, representing a polymer as a sphere is far more tenable, and one expects only quantitative differences between the PSFV and PRISM approaches for ideal coils. This argument is verified in Sec. IV C.

\section{Athermal limit}

Under athermal solvent conditions, strong polymerpolymer repulsions result in qualitatively new phase behavior associated with a nonzero value of $\widetilde{C}_{p p}^{0}$. The latter is self-consistently chosen to enforce local excluded volume between polymer segments: $g_{p p}(r=0)=0$. This constraint is highly nontrivial and effectively couples local packing considerations to long wavelength (thermodynamic) properties. It is the origin of the ability of PRISM theory for athermal semidilute polymer solutions to correctly capture strong correlation effects consistent with scaling arguments and field theory. ${ }^{14,24}$ The analytic result in the low polymer concentration limit is 9

$$
-\hat{C}_{p p}^{0} \propto \frac{l_{p}^{4}}{\xi R}\left(\frac{1}{1-\phi_{s}}+\frac{3 \xi \phi_{s}\left(2+\gamma_{1}\right)^{2}}{2 \sqrt{2} \gamma_{1}^{2}\left(1-\phi_{s}\right)^{2}}\right),
$$


where $l_{p}^{2}=R_{g}^{2} / 2 N$ is an effective polymer step or persistence length ( $N$-independent for ideal coils and $l_{p}^{2} \propto N^{1 / 5}$ for selfavoiding walks). In the dilute colloid limit, $-\hat{C}_{p p}^{0} \propto R_{g}^{-1}$, consistent with recovering $B_{2, p p} \propto R_{g}^{3}$ from Eq. (15) for both ideal and good solvent conditions. With increasing colloid volume fraction, $-\hat{C}_{p p}^{0}$ and the corresponding contribution to the polymer second virial coefficient in Eq. (15) become increasingly repulsive, approaching a finite limiting value in the extreme nanoparticle limit.

Any approximation which computes $B_{2, p p}$ by simply adding a "bare" isolated two chain contribution (second virial in absence of colloids) to the colloid-induced contribution determined under ideal conditions [Eq. (21)] is not valid. This is dramatically illustrated by the fact Eqs. (15) and (20) imply that in the nanoparticle regime the PSFV approach predicts $B_{2, p p} \sim-R_{g}^{4} / R$, which corresponds to a much greater depletion attraction than the PRISM result of $B_{2, p p}$ $\sim R_{g}^{3} .{ }^{9}$ The full PRISM theory polymer second virial coefficient result in the $R_{g} \gg R$ regime is ${ }^{9}$

$$
B_{2, p p}=\frac{\sqrt{2} \pi R_{g}^{3}}{\left(1+2 \phi_{s}\right)}\left[1-\frac{36 \phi_{s}}{1-\phi_{s}} \frac{1+\gamma_{1}+\gamma_{1}^{2} / 3}{\gamma_{1}^{3}}\right], \quad \xi \gg 1 .
$$

The $B_{2, p p} \sim R_{g}^{3}$ dependence is identical to the pure polymer solution behavior, in which case the prefactor is proportional to the segmental excluded volume parameter, which is positive in good solvents. Colloidal particles induce polymer clustering which reduces, and can (depending on colloid volume fraction) reverse the sign of, the polymer effective second virial coefficient. In the extreme colloid limit of $\xi \ll 1$, very different behavior is predicted by PRISM theory ${ }^{9}$

$$
B_{2, p p}=-\frac{\pi}{12} D^{3} \frac{\phi(1-\phi)^{2}}{(1+2 \phi)^{2}}, \quad \xi \ll 1 .
$$

The magnitude of the polymer second virial coefficient is now controlled by colloid size and is negative for all colloid volume fractions. A detailed study of $B_{2, p p}$ over the entire range of parameters has been given previously. ${ }^{9}$

As will be shown in Sec. IV B, the $\xi$ - and $\phi_{s}$-dependent consequences of polymer-polymer repulsions on $B_{2, p p}$ is the fundamental physical effect which reverses in good solvents the (incorrect) shifts of phase boundaries with $R_{g} / R$ predicted by the PSFV and related classic approaches. A prior attempt within the PSFV framework to access the role of polymer-polymer repulsions concluded it had only a minor effect on phase behavior, ${ }^{34}$ in contrast to PRISM theory and experiment. ${ }^{4}$ The primary origin of this disagreement is the inability of the prior approach to determine how $B_{2, p p}$ is modified by the presence of colloids under conditions of strong polymer repulsions.

\section{RESULTS}

\section{A. Role of nondilute polymer concentration for athermal solvents}

"Full" (contributions through all orders in $c_{p}$ ) PRISMmPY numerical results for polymer-colloid mixture spinodal boundaries in an athermal good solvent have been presented

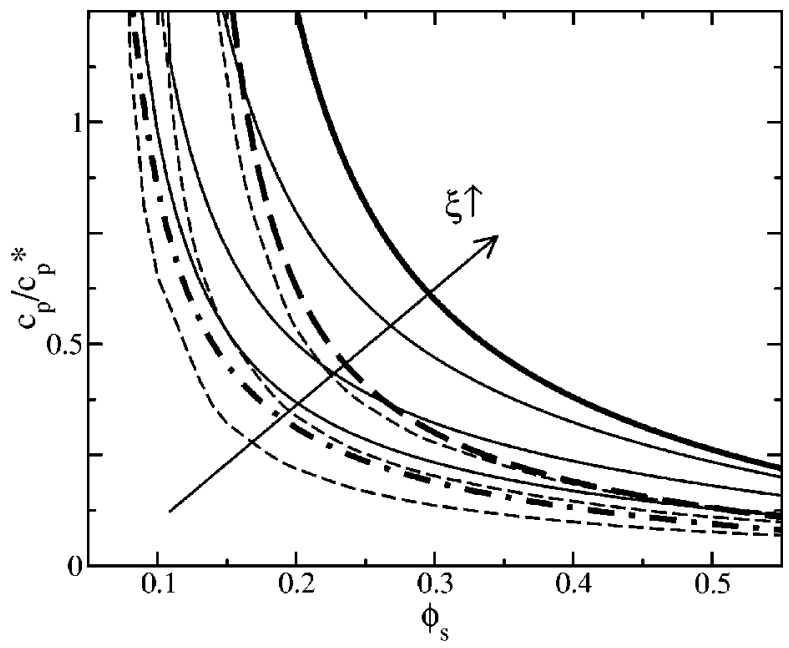

FIG. 1. PRISM-mPY spinodal boundaries for an athermal solvent and $\xi$ $=0.14,0.56,2.83$. Perturbative PRISM results are shown as thin dashed lines, the extreme nanoparticle limit is the thick dashed line. The full PRISM results are shown as thin solid lines, the extreme nanoparticle limit is the top thick solid lines, and the extreme colloid limit is the thick dot-dashed line.

previously. ${ }^{8-10}$ Analytic expressions for the spinodal have been derived in the extreme $\xi \rightarrow 0$ and $\xi \rightarrow \infty$ limits, ${ }^{8,10}$ and are given by

$$
\begin{aligned}
& \frac{c_{p}^{+}}{c_{p}^{*}}=\frac{2 \sqrt{2}}{3} \frac{\left(1+\phi_{s}-2 \phi_{s}^{2}\right)}{22 \phi_{s}-1}, \quad \xi \rightarrow 0 \quad \mathrm{mPY}, \\
& \frac{c_{p}^{+}}{c_{p}^{*}}=\frac{4 \sqrt{2}}{3} \frac{\left(1+2 \phi_{s}\right)\left(1-\phi_{s}\right)}{\phi_{s}\left(6 \gamma_{1}-1\right)-2}, \quad \xi \rightarrow \infty \quad \mathrm{mPY} .
\end{aligned}
$$

PRISM-mPY predicts no spinodal demixing at low enough $\phi_{s}$ in athermal solvents. As observed from Fig. 1, the spinodal boundaries shift monotonically to higher $c_{p} / c_{p}^{*}$ as $R_{g} / R$ increases. ${ }^{8,10}$

Our present interest is to see if the $\mathrm{O}\left(c_{p}\right)$ version of PRISM predictions for the spinodal are qualitatively the same as those of full PRISM theory. As discussed in Sec. III, when $\xi \gg 1$ the first order correction $\Delta \hat{C}_{s s}$ is negligible and Eq. (19) applies. By using Eqs. (9), (11), and (24) in Eq. (19), straightforward algebra yields an analytic expression for $c_{p} / c_{p}^{*}$ along the spinodal curve. Remarkably, we find the result is exactly a factor of 2 below the full theory result of Eq. (27)! Hence, in the nanoparticle limit, there is only a modest quantitative correction due to all the higher order in polymer concentration contributions which is independent of sphere volume fraction. The lower values of reduced polymer concentration at the spinodal is expected, since polymer-polymer repulsions stabilize the mixed phase. ${ }^{13,28}$

Comparison of the full and $\mathrm{O}\left(c_{p}\right)$ spinodal boundaries is shown in Fig. 1 over a range of polymer-to-colloid size ratios. The qualitative trend of monotonically increasing fluidfluid miscibility with increasing $\xi$ is established even at the simple $\mathrm{O}\left(c_{p}\right)$ level. Differences between the perturbative and full theories are a factor of 2 or less under all conditions studied. The influence of higher order polymer-polymer repulsions does increase with colloid volume fraction. This makes physical sense since the ability of coils to avoid each 


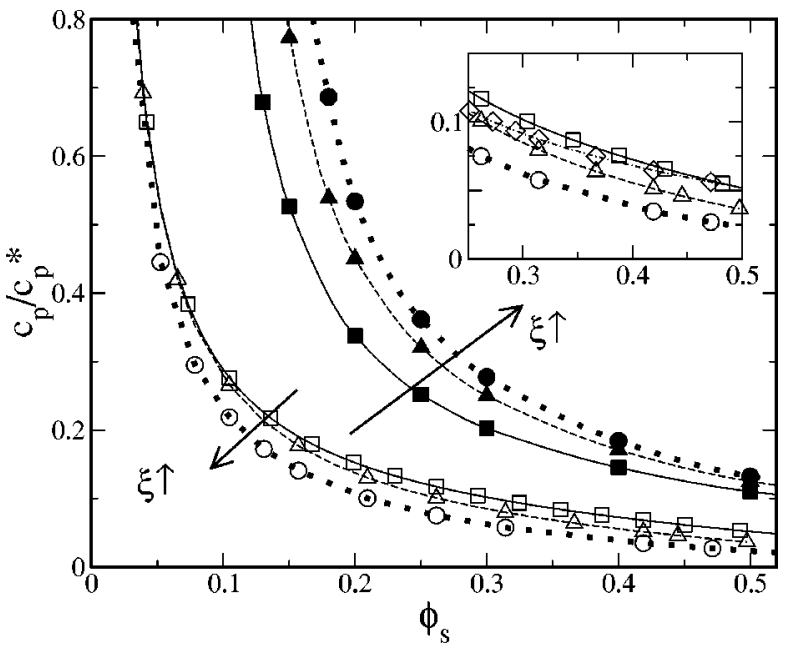

FIG. 2. Perturbative PRISM-mPY spinodal boundaries under athermal good solvent conditions (solid symbols) and ideal solvent conditions (empty symbols) for $\xi=0.56$ (squares), 1.41 (triangles), and 2.83 (circles). The inset shows only the spinodals under ideal solvent conditions at high colloid volume fraction, with the addition of the $\xi=0.14$ (diamonds) case.

other in the free volume between particles decreases as $\phi_{s}$ increases. Regarding the incorrect spinodal predictions of the PSFV approach, we can definitively conclude it does not fundamentally arise from higher order polymer-polymer interactions or strong modification of colloidal structure and free volume by depletion forces.

\section{B. Role of solvent quality: Ideal versus athermal}

Having established the quality of $\mathrm{O}\left(c_{p}\right)$ PRISM predictions compared with the full theory, we employ the numerically and analytically simpler lowest order method to further investigate the role of polymer-polymer interactions. $\mathrm{O}\left(c_{p}\right)$ spinodal predictions for the athermal good solvent and ideal solvent cases are compared in Fig. 2. The primary trends are as follows. (i) At fixed $\xi$ and $\phi_{s}$, fluid-fluid demixing under ideal solvent conditions always occurs at lower reduced polymer concentration than in good solvents. The reduction factor is $\sim 3-7$ (over a wide a range of $\phi_{s}$ ), with deviations growing as $R_{g} / R$ increases. (ii) At very high colloid volume fractions, the ideal coil spinodals do shift down with increasing size asymmetry $\xi$. However, in contrast with the athermal solvent behavior, at low and moderate colloid volume fractions the spinodal boundaries show a surprising nonmonotonic shifting, or near constancy, as polymers increase in size relative to colloids. This nonmonotonicity becomes more pronounced with decreasing colloid volume fraction, but is still weakly present up to $\phi_{s} \sim 0.35-0.4$. For $\xi<1$, the spinodals first shift towards higher reduced polymer concentration (improved miscibility) as $\xi$ increases, and then reverse with further increase of $\xi$. Hence spinodal "curve crossings" are predicted. This unexpected behavior has been recently found in $\mathrm{AO}$ model simulations ${ }^{17}$ of an ideal polymer-colloid system. Thus, $\mathrm{O}\left(c_{p}\right)$ PRISM-mPY theory captures a very subtle feature of ideal solvent phase behavior. Preliminary experimental and full mPY numerical calculations reveal an even stronger, $\phi_{s}$-dependent, nonmonotonic variation of demixing boundaries. ${ }^{35}$

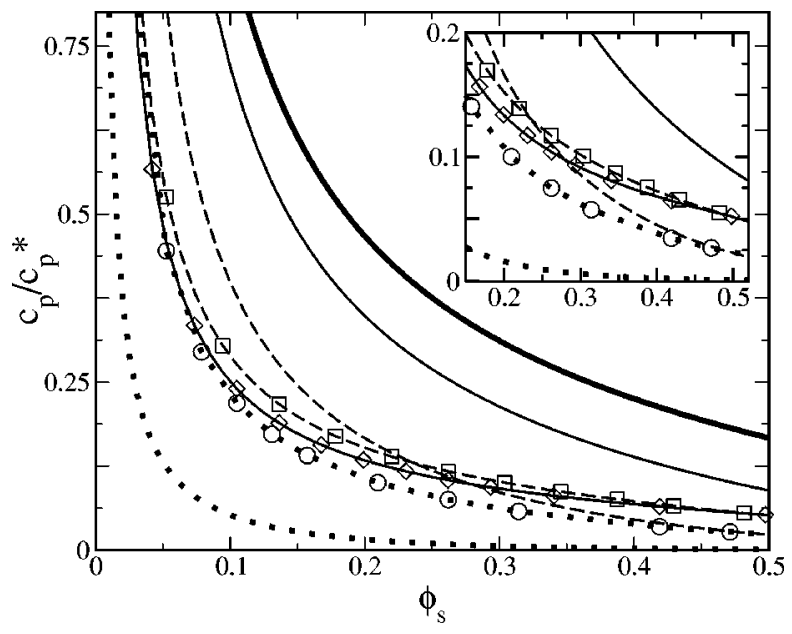

FIG. 3. PSFV and perturbative PRISM-mPY spinodal boundaries under ideal solvent conditions. PRISM-mPY results are shown for $\xi=0.14$ (thin solid line with diamonds), 0.56 (dashed line with squares), 2.83 (dotted line with circles), and the corresponding PSFV predictions for the same ratios are shown without symbols. The thick solid line is the PSFV prediction in the extreme colloid limit. The inset shows the dense colloid regime in more detail.

Since direct polymer-polymer interactions are absent in ideal solvents, the more complex response of the spinodals to size asymmetry and particle volume fraction must arise from a competition between polymer-sphere and sphere-sphere direct correlations. As shown in Sec. III, when the polymers are comparable in size or larger than the particles, an increase of $R_{g}$ has less and less influence on local colloidal structure and the $\Delta \hat{C}_{s s}$ contribution becomes negligible relative to $\widetilde{C}_{p s}^{0}{ }^{2}$. Thus, the spinodal boundary is dominated by polymer-particle correlations (insertion chemical potential) and simplifies to: $c_{p}^{+} / c_{p}^{*} \sim 1 /\left(\widetilde{C}_{p s}^{0}{ }^{2} c_{p}^{*}\right)$. Since $\widetilde{C}_{p s}^{0} \sim R_{g}^{4}$ for $\xi \gg 1$ and $1 / c_{p}^{*} \sim R_{g}^{3}$, the spinodal curve shifts down as $\xi$ grows. Using these results and Eqs. (9) and (22) yields the explicit result

$$
c_{p}^{+} / c_{p}^{*}=0.17\left(1-\phi_{s}\right)^{2} /\left(\xi \phi_{s}\right), \quad \text { ideal } \mathrm{mPY} 0, \quad \xi \rightarrow \infty,
$$

where the notation $\mathrm{mPY} 0$ refers to the lowest order $\mathrm{mPY}$ PRISM theory. Equation (29) quantifies the scaling results shown earlier in Eq. (23).

In the $\xi \ll 1$ colloidal regime, Eqs. (16) and (20) imply $c_{p}^{+} / c_{p}^{*} \sim 1 /\left(D^{3} \phi_{s}\left(\widetilde{C}_{p s}^{2}+\Delta \hat{C}_{s s}\right) c_{p}^{*}\right)$. Since $\widetilde{C}_{p s}^{0}{ }^{2} \sim-\Delta \hat{C}_{s s}$ $\sim R^{6}$, we find that low-order analytic expansions in $\xi$ do not suffice to establish the qualitative trends due to cancellations. Numerical calculations are required, and Fig. 2 shows the spinodals shift up as $\xi$ increases in the colloid regime, opposite to the nanoparticle regime behavior. Hence, a reversal of the qualitative shifting of the demixing curves with size asymmetry in ideal solvents may be understood as a subtle consequence of the change in scaling of the $s-s$ and $s-p$ direct correlation functions with polymer and particle sizes.

\section{Role of conformational entropy in ideal solvents}

The $\mathrm{O}\left(c_{p}\right)$ PRISM-mPY predictions for ideal coils are compared to PSFV theory in Fig. 3. This comparison prima- 
rily reveals the role of treating a polymer as a connected chain with segmental conformational degrees of freedom. In contrast to the complex nonmonotonic behavior of the PRISM-mPY spinodals, the PSFV theory predicts phase separation boundaries shift monotonically downward with increasing $\xi$. Hence, one can deduce the crucial importance of treating a polymer as an open interpenetrating fractal object and not an impenetrable hard sphere. The PRISM spinodals also shift far less strongly with increasing size asymmetry (a factor of $\sim 2$ for a factor of 20 change in $\xi$ ), compared to a much larger factor of $\sim 10-20$ by the PSFV model which is due to the increasing overestimation of the polymer insertion chemical potential (or depletion attraction between particles) as $\xi$ increases.

It is also interesting to note that the PSFV model spinodals are above their PRISM analogs for small polymers, but shift to well below for long polymers. This systematic difference has been previously documented in a combined theory-experiment study of colloid concentration fluctuations in athermal solvents. ${ }^{18}$ The interpretation advanced was that for small polymers the PSFV model underpredicts depletion attraction, since it ignores the additional conformational entropy gained by polymers leaving the spatial region near or between colloids. But, for large polymers the PSFV representation of a chain as a hard sphere ignores the ability of polymers to "wrap around" small particles, resulting in a strong overestimation of depletion attraction. Such competing effects imply the existence of a narrow range of $\xi$ values $(\sim 0.5)$ where the PSFV and PRISM predictions cross due to a cancellation of errors. Such "agreement" is fortuitous.

\section{Influence of nonlocal entropic repulsion in ideal solvents}

Analytic results for the spinodal boundaries of mixtures of ideal coils and hard spheres have been previously derived using the local PY approximation $(\lambda=0)$. Comparison of the PY and mPY predictions allows the influence of nonlocal entropic repulsion to be accessed within the common PRISM framework. In athermal solvents, the consequences are enormous since PY (incorrectly) predicts miscibility under all conditions due to its quantitative underprediction of depletion attraction. ${ }^{8,26,36}$ However, for ideal coils one expects nonlocal entropic effects result in relatively modest corrections since phase separation of atomic mixtures is predicted by PY type theories under such strongly nonadditive excluded volume conditions. ${ }^{37}$

Example comparisons of $\mathrm{O}\left(c_{p}\right)$ spinodals are shown in Fig. 4. The PY demixing curves always lie above their mPY counterparts due to the extra nonlocal entropic driving force for phase separation. The PY theory predicts a much stronger dependence on size asymmetry than the mPY. This is due to the reduction in importance of nonlocal entropic effects as $\xi$ increases. ${ }^{8,10}$ A consequence of the latter behavior is that the PY and mPY spinodals disagree less with increasing $\xi$, and become identical in form as $\xi \rightarrow \infty$ limit (see below).

The trends suggested by the numerical results in Fig. 4 can be understood based on analytic analysis. The full (all orders in $c_{p}$ ) ideal coil PY spinodal is given by ${ }^{9,36}$

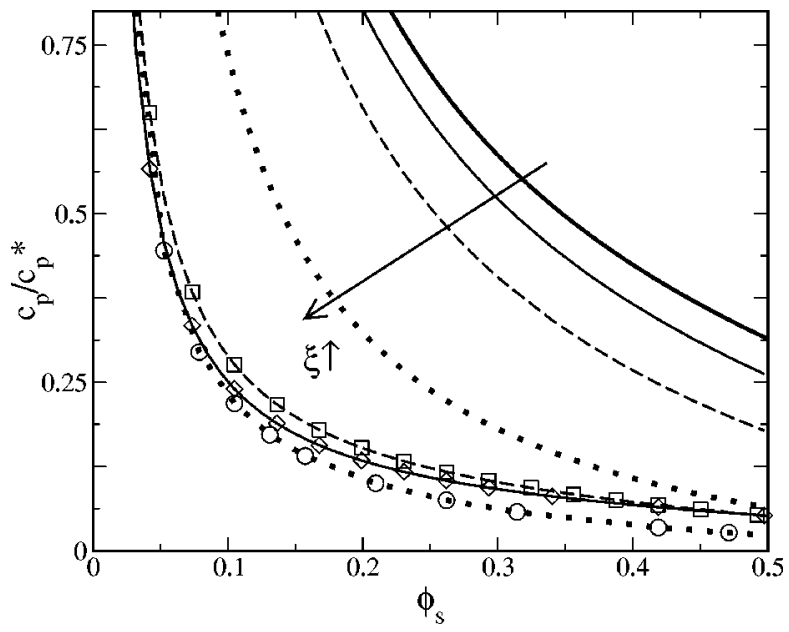

FIG. 4. Perturbative PRISM-PY and -mPY spinodal boundaries for ideal solvent conditions. The mPY results are shown for $\xi=0.14$ (thin solid line with diamonds), 0.56 (dashed line with squares), 2.83 (dotted line with circles), and the PY results for the same ratios are shown without the symbols. The thick solid line is the PY curve in the extreme colloid limit.

$$
\frac{c_{p}^{+}}{c_{p}^{*}}=\frac{\xi}{9} \frac{\left(1+2 \phi_{s}\right)^{2}}{\phi_{s}}\left[-1+\sqrt{1+\frac{8}{\xi^{2}}\left(\frac{1-\phi_{s}}{1+2 \phi_{s}}\right)^{2}}\right],
$$

which in the extreme limits simplifies to

$c_{p}^{+} / c_{p}^{*}=2 \sqrt{2}\left(1-\phi_{s}\right)\left(1+2 \phi_{s}\right) /\left(9 \phi_{s}\right), \quad \xi \rightarrow 0, \quad \mathrm{PY}$

$c_{p}^{+} / c_{p}^{*}=(4 / 9)\left(1-\phi_{s}\right)^{2} /\left(\xi \phi_{s}\right), \quad \xi \rightarrow \infty, \quad$ PY.

The corresponding $\mathrm{O}\left(c_{p}\right)$ PRISM-PY(PY0) spinodal boundary can also be deduced ${ }^{9,35}$

$$
\frac{c_{p}^{+}}{c_{p}^{*}}=\frac{\sqrt{2}}{9} \frac{\left(1-\phi_{s}\right)^{2}\left(1+2 \phi_{s}\right)}{\phi_{s}\left(1-\phi_{s}\right)+\phi_{s}\left(1+2 \phi_{s}\right) \xi /(2 \sqrt{2})}, \quad \text { PY } 0 .
$$

The limiting $\mathrm{O}\left(c_{p}\right)$ PY and PSFV spinodal boundaries are given by

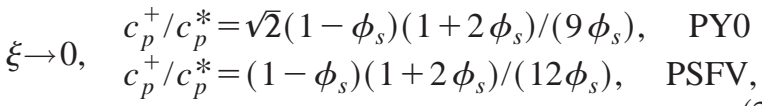

$$
\begin{aligned}
& \begin{array}{ll}
\xi \rightarrow \infty, & c_{p}^{+} / c_{p}^{*}=(4 / 9)\left(1-\phi_{s}\right)^{2} /\left(\xi \phi_{s}\right), \quad \text { PY0 } \\
& c_{p}^{+} / c_{p}^{*}=\left(1-\phi_{s}\right)^{4} /\left[\xi^{3} \phi_{s}\left(1+2 \phi_{s}\right)^{2}\right], \quad \text { PSFV } .
\end{array}
\end{aligned}
$$

There are multiple interesting aspects of these analytic results. Consider first the relationship between mPY and PY which reveals the role of nonlocal conformational entropy. (i) In contrast to the mPY prediction for ideal solvents, the full and $\mathrm{O}\left(c_{p}\right)$ PY results of Eqs. (30) and (33) predict that as $\xi$ increases the spinodals shift monotonically downward (to lower $c_{p} / c_{p}^{*}$ ) for all colloid volume fractions. Thus, the subtle nonmonotonic behavior found in the $\mathrm{mPY}$ theory is a consequence of nonlocal entropic restrictions on polymer conformational degrees of freedom near a hard particle. (ii) 
The PY spinodal approaches zero as $\xi^{-1}$ for large size asymmetry $(\xi \rightarrow \infty)$, in agreement with PRISM-mPY in Eq. (29). (iii) The full and perturbative PY spinodals become qualitatively identical in the large polymer limit. This is generically expected for all theories for ideal coils since as size asymmetry increases and the spinodal curves monotonically decrease towards zero, a lowest order in polymer concentration treatment becomes exact. (iv) The $\mathrm{O}\left(c_{p}\right)$ PY and mPY spinodals of Eqs. (35) and (29) are identical in form in the $\xi$ $\rightarrow \infty$ limit, differing by a numerical prefactor $\sim 0.38$. This is presumably a consequence of the nonlocal entropic effects having an intrinsic spatial range $\sim D$ in this limit, ${ }^{9}$ which becomes increasingly less important as $\xi$ grows. Quantitatively, the mPY spinodal is lower than its PY analog due to the nonlocal entropy consideration.

Concerning connections between the PSFV and PRISM approaches we conclude the following. (v) As $\xi \rightarrow \infty$, PRISM-PY predicts the same qualitative trend as PSFV theory in the sense that the spinodals of the latter also shift downward in an unbounded and monotonic manner as $\xi$ increases. However, the PSFV approach predicts the spinodals approach zero as $1 / \xi^{3}$, qualitatively much faster than the PRISM-PY (or $\mathrm{mPY}$ ) prediction of $1 / \xi$. This difference reveals the large consequences of the "polymer $\approx$ phantom sphere" approximation. (vi) Remarkably, in the $\xi \rightarrow 0$ limit the $\mathrm{O}\left(c_{p}\right)$ PY and PSFV approaches predict spinodal boundaries of exactly the same mathematical form [Eq. (34)], differing only by a modest numerical prefactor of $\sim 2$. Perhaps this is because for small "pointlike" polymers the internal conformational degrees of freedom are not qualitatively important, and large colloids obviously cannot penetrate the polymer coil domain. Quantitatively, the PSFV spinodal lies between the two integral equation theory curves. When $\xi$ $\ll 1$, the colloids become wall-like relative to the polymers, and neither PRISM-PY nor PSFV approaches account for the loss of polymer conformational entropy near a hard surface.

\section{CONCLUSIONS}

We summarize our findings by returning to the issues raised in the Introduction.

(1) For athermal good solvents, analysis of the lowest order in polymer concentration version of PRISM-mPY shows that the prior novel prediction ${ }^{8}$ of increased miscibility with increasing $R_{g} / R$ is not fundamentally tied to physical mesh formation nor strong polymer-induced colloid clustering. This is consistent with recent numerical PRISM-mPY studies of polymer-particle and polymerpolymer structural correlations, which show a remarkably weak dependence on polymer concentration. ${ }^{10}$ The key is a proper treatment of the polymer second virial coefficient $B_{2, p p}$, which contains direct and colloidinduced contributions, both of which depend on size asymmetry and particle volume fraction reflecting the importance of how polymer chains organize in the empty space between particles. ${ }^{9}$ The qualitative error incurred by the classic theories ${ }^{7,11}$ has been shown to arise from neglect of this aspect. This conclusion is consistent with the prior demonstration that use of the PRISM-mPY in- sertion chemical potential in the PSFV theory has no qualitative consequences on the (incorrect) spinodal behavior. $^{4}$

(2) The differences between athermal solvent spinodals predicted by the full and lowest order PRISM theories are modest, a factor $\sim 2$ or less for all parameters. Higher order contributions in polymer concentration stabilize the miscible phase in both athermal and ideal solvents, and the common physical reason is clear. Increasing polymer concentration enhances the depletion attraction between particles. This results in physical clustering of the particles, and hence more "free volume" in the mixture. The latter reduces the free-energy cost of adding more polymers, and hence delays phase separation.

(3) For ideal theta solvent conditions, the phase separation behavior predicted by PRISM-mPY theory (at the simplest perturbative level) is completely different for all size asymmetries and colloid volume fractions than found for athermal solvents. Hence, polymer-polymer repulsions have remarkably large consequences. For $R_{g}$ $\gg R$, the suspension miscibility does worsen with increasing size asymmetry, opposite to the athermal solvent behavior. However, intriguing and complex subtleties are also predicted. Over a significant range of intermediate size asymmetries the spinodal curves are either nearly constant and/or display a nonmonotonic shifting (at lower $\phi_{s}$ ) as $\xi$ is varied. This behavior arises due to a competition between polymer-particle and particle-particle correlation contributions to the demixing condition which scale in different manners with size asymmetry. Major differences between the PRISM predictions and those of the PSFV model are generally found for all values of $\xi$ and $\phi_{s}$. The deviations increase with colloid volume fraction, and are systematic in size asymmetry in that weaker (much stronger) depletion effects are predicted by the PSFV model than PRISM theory in the colloid (nanoparticle) regimes.

(4) By comparing the PY and MPY versions of PRISM theory the role of nonlocal conformational entropic effects was established under ideal solvent conditions. Qualitatively identical spinodal behavior is predicted in the extreme nanoparticle limit, and is also expected in the colloid limit, in sharp contrast to the athermal solvent case. This is due to the highly nonadditive nature of excluded volume effects if polymer-polymer interactions are ignored. However, the PY theory predicts a monotonic reduction of miscibility with increasing $\xi$, and thus misses the nonmonotonic variations predicted by $\mathrm{mPY}$ and also seen in simulation. ${ }^{17}$ Hence, nonlocal conformational entropy effects appear essential to capture the subtle aspects of ideal solvent phase behavior.

Full numerical mPY theory calculations are reported elsewhere, along with complementary experimental phase diagram and turbidity experiments. ${ }^{35}$ The curious nonmonotonic shifting of fluid-fluid boundaries predicted by perturbative mPY PRISM theory is enhanced when higher order polymer concentration contributions are included.

PRISM is fundamentally a theory of structure. Predic- 
tions for how site-site radial distribution functions and scattering structure factors depend on solvent quality are available and will be reported in future work. Such structural information is essential input to microscopic dynamical approaches such as mode-coupling theory. ${ }^{30,38}$

Finally, the present work has two additional implications. First, the demonstration that a perturbative PRISMmPY theory is quite accurate compared to the full (much more numerically intensive) version of the theory may provide a computationally convenient basis for calculation of fluid-fluid and fluid-crystal binodals. Second, understanding the two extreme limits of athermal and ideal solvents provides a foundation to treat the general solvency situation, which promises to display even more subtle and complex phase behavior.

\section{ACKNOWLEDGMENTS}

Work at Illinois was supported by the Nanoscale Science and Engineering Initiative of the National Science Foundation under NSF Award Number DMR-0117792. M.F. was supported by the Deutsche Forschungsgemeinschaft under Grant No. Fu309/3 and through the SFB 563. Many stimulating discussions with Syed Ali Shah and Chip Zukoski are gratefully acknowledged.

\section{APPENDIX: NUMERICAL METHODS}

The full PRISM-mPY matrix Eqs. (3) and (6) are solved numerically using a Picard iteration scheme with a mixing parameter to improve convergence. ${ }^{14}$ The central iterand is the quantity $\Gamma_{i j}=h_{i j}-C_{i j}$. However, use of the field theoretic thread polymer model $\left[\widetilde{C}_{p p}(k)=C_{p p}\right]$ introduces modifications to the iteration scheme which makes convergence more difficult. Since $C_{p p}$ is a constant, a Newton-Raphson $(\mathrm{N}-\mathrm{R})$ routine is embedded in the Picard iteration to enforce the thread $\left[g_{p p}(r=0)\right]$ constraint for simultaneous calculations of the coupled functions $\widetilde{C}_{p p}(k), \hat{C}_{s s}(k)$, and $\widetilde{C}_{p s}(k)$.

In the $\mathrm{O}\left(c_{p}\right)$ version of PRISM-mPY, the PRISM equations may be decoupled and solved independently in the order of sphere-sphere, polymer-sphere, and polymerpolymer integral equations. $\hat{C}_{s s}\left(\rho_{p} \rightarrow 0\right)$ and $\widetilde{C}_{p s}\left(\rho_{p} \rightarrow 0\right)$ are calculated using the Picard method, and $C_{p p}$ is determined from the N-R method. Once these functions are found, the first-order correction term $\Delta \hat{C}_{s s}$ is then determined using the Picard method.

In order to calculate the spinodal boundaries, highly accurate estimates of the values of $C_{p p}, \hat{C}_{s s}(k=0), \widetilde{C}_{p s}(k$ $=0), \Delta \hat{C}_{s s}(k=0)$ are required because the solution of Eq. (8) is very sensitive to numerical subtraction errors. The latter are reduced and characterized by (i) varying the error tolerance defined for convergence, and (ii) varying the grid (spacing and system size) on which the calculations are performed. A four-point Lagrange interpolation method is utilized to compute the $k=0$ values, and these errors are negligible compared to the inherent errors of the numerical algorithm. The overall errors of the numerical calculations are benchmarked by comparison to the (semi)analytic results given for the athermal good solvent mixture. ${ }^{8,10}$ The fully numerically determined spinodals are found to overestimate by less than $5 \%$ the (semi)analytic spinodals for $R_{g} / R$ $=0.14-2.83$, with the errors largest at high $c_{p}$ and $R_{g} / R$.

${ }^{1}$ J. N. Israelachvili, Intermolecular and Surface Forces (Academic, London, 1991); W. B. Russel, D. A. Saville, and W. R. Schowalter, Colloidal Dispersions (Cambridge University Press, Cambridge, 1991).

${ }^{2}$ W. C. K. Poon, J. Phys.: Condens. Matter 14, R859 (2002).

${ }^{3}$ S. M. Ilett, A. Orrock, W. C. K. Poon, and P. N. Pusey, Phys. Rev. E 51, 1344 (1995).

${ }^{4}$ S. Ramakrishnan, M. Fuchs, K. S. Schweizer, and C. F. Zukoski, J. Chem. Phys. 116, 2201 (2002).

${ }^{5}$ W. Poon, P. Pusey, and H. N. W. Lekkerkerker, Phys. World 1996, 27.

${ }^{6}$ E. H. A. de Hood, W. K. Kegel, A. van Blaaderen, and H. N. W. Lekkerkerker, Phys. Rev. E 64, 021407 (2001).

${ }^{7}$ H. N. W. Lekkerkerker, W. C. K. Poon, P. N. Pusey, A. Stroobants, and P. B. Warren, Europhys. Lett. 20, 559 (1992)

${ }^{8}$ M. Fuchs and K. S. Schweizer, Europhys. Lett. 51, 621 (2000).

${ }^{9}$ M. Fuchs and K. S. Schweizer, Phys. Rev. E 64, 021514 (2001).

${ }^{10}$ M. Fuchs and K. S. Schweizer, J. Phys.: Condens. Matter 14, R239 (2002).

${ }^{11}$ A. P. Gast, C. K. Hall, and W. B. Russel, J. Colloid Interface Sci. 96, 251 (1983).

${ }^{12}$ S. Asakura and F. Oosawa, J. Chem. Phys. 22, 1255 (1954); J. Polym. Sci. 33, 183 (1958).

${ }^{13}$ J. F. Joanny, L. Leibler, and P. G. de Gennes, J. Polym. Sci., Polym. Phys. Ed. 17, 1073 (1979).

${ }^{14}$ K. S. Schweizer and J. G. Curro, Adv. Chem. Phys. 98, 1 (1997), and references cited therein.

${ }^{15}$ K. S. Schweizer and J. G. Curro, Adv. Polym. Sci. 116, 319 (1994).

${ }^{16}$ D. Chandler, Studies in Statistical Mechanics (North-Holland, Amsterdam, (1982), Vol. VIII, p. 274; D. Chandler and H. C. Andersen, J. Chem. Phys. 57, 1930 (1972)

${ }^{17}$ P. G. Bolhius, A. A. Louis, and J. P. Hansen, Phys. Rev. Lett. 89, 128302 (2002).

${ }^{18}$ S. Ramakrishnan, M. Fuchs, K. S. Schweizer, and C. F. Zukoski, Langmuir 18, 1082 (2002).

${ }^{19}$ R. Tuinier, G. A. Vliegenhart, and H. N. W. Lekkerkerker, J. Chem. Phys. 113, 10768 (2000).

${ }^{20}$ M. Schmidt, H. Löwen, J. M. Brader, and R. Evans, Phys. Rev. Lett. 85, 1934 (2000).

${ }^{21}$ R. P. Sear, Phys. Rev. Lett. 86, 4696 (2001).

${ }^{22}$ E. J. Meijer and D. Frenkel, J. Chem. Phys. 100, 6873 (1994).

${ }^{23}$ H. Reiss, H. L. Frish, and J. L. Lebowitz, J. Chem. Phys. 31, 369 (1959); J. L. Lebowitz, E. Helfand, and E. Praestgaard, ibid. 43, 133 (1964).

${ }^{24}$ M. Doi and S. F. Edwards, Theory of Polymer Dynamics (Oxford University Press, Oxford, 1986); P. G. deGennes, Scaling Concepts in Polymer Physics (Cornell University Press, Ithaca, 1979).

${ }^{25}$ For athermal solvent conditions, the Gaussian polymer model of Eq. (5) is adopted as a technical approximation to a self-avoiding walk polymer since it allows major analytic progress to be made in solving the integral equations using the Baxter factorization technique (Refs. 8-10).

${ }^{26}$ J. L. Lebowitz and J. S. Rowlinson, J. Chem. Phys. 41, 133 (1964); J. L. Lebowitz, Phys. Rev. 133, A895 (1964).

${ }^{27}$ A. Vrij, J. Chem. Phys. 69, 1742 (1978).

${ }^{28}$ A. P. Chatterjee and K. S. Schweizer, J. Chem. Phys. 109, 10464 (1998); 109, 10477 (1998).

${ }^{29}$ The ideal polymer model employed is a literal one in that polymer segments can occupy the same space in a given configuration. Such a toy model has been widely employed in prior theoretical and simulation work since it significantly reduces the technical complexity of the problem. In reality, theta conditions require the presence of both repulsive and attractive segment-segment interactions which perfectly cancel at the second virial coefficient level (Ref. 24).

${ }^{30}$ J. P. Hansen and I. R. McDonald, Theory of Simple Liquids, 2nd ed. (Academic, London, 1986).

${ }^{31}$ J. G. Kirkwood and F. P. Buff, J. Chem. Phys. 19, 774 (1951). Although the Maxwell relation may be invoked to calculate $\Delta \hat{C}_{s s}(k=0)$, it is well known not to be exactly obeyed by integral equation theories (Refs. 30, $36)$. We find for the present problem that the quantitative differences between the $\Delta \hat{C}_{s s}(k=0)$ determined from numerically solving the integral 
equations versus deduced using the Maxwell relation of Eq. (17) are less than $10 \%-15 \%$. However, calculation of the spinodal boundary from Eq. (4) or (8) can be extremely sensitive to quantitative accuracy of the direct correlation functions. This is especially true in the extreme colloid limit where accurate calculation of the order $\xi^{2}$ correction to $\Delta \hat{C}_{s s}(k=0)$ plays a critical role in spinodal predictions. Hence, in all our prior (Refs. 8-10) and present work the Maxwell relation is not employed in the calculation of spinodals.
${ }^{32}$ R. Tuinier, G. A. Vliegenthart, and H. N. W. Lekkerkerker, J. Chem. Phys. 113, 10768 (2000).

${ }^{33}$ M. Schmidt and M. Fuchs, J. Chem. Phys. 117, 6308 (2002).

${ }^{34}$ P. B. Warren, S. B. Ilett, and W. C. K. Poon, Phys. Rev. E 52, 5205 (1995).

${ }^{35}$ S. A. Shah, Y.-L. Chen, K. S. Schweizer, and C. F. Zukoski, J. Chem. Phys. (in press).

${ }^{36}$ M. Fuchs and K. S. Schweizer (unpublished).

${ }^{37}$ D. Gazzillo, J. Chem. Phys. 95, 4565 (1991).

${ }^{38}$ W. Gotze and L. Sjogren, Rep. Prog. Phys. 55, 241 (1992). 